\title{
Hot Atom Damage (HAD) Limited TDDB Lifetime of Ferroelectric Memories
}

\author{
Muhammad Masuduzzaman* and Muhammad Ashraful Alam ${ }^{+}$ \\ "Email: masuduzzaman@ieee.org, ${ }^{+}$Email: alam@purdue.edu, Phone: 765-494-5988, Fax: 765-494-2706 \\ Department of ECE, Purdue University, West Lafayette, IN 47907, USA
}

\begin{abstract}
The AC/DC dielectric lifetime (TDDB) of ferroelectric materials have traditionally been interpreted similar to a classical gate oxide. In this paper, we demonstrate a fundamentally different kinetic mechanism of damage involving hot atom (HAD) in ferroelectric materials to interpret the severe reduction of AC lifetime, coupled with the counterintuitive increase in the Weibull slope. We show that, beyond a critical operating condition, the atoms at the domain walls are heated by the AC field, as they shuttle across the double-well energy landscape of such materials. An elegantly simple analytical model (i) interprets critical TDDB experiments, (ii) suggests strategies (e.g., pulse shaping) to minimize HAD significantly, and (iii) predicts device lifetime at arbitrary operating conditions.
\end{abstract}

\section{Introduction}

Dielectric materials with memory functionalities have recently gained interest in much broader area of applications. This includes emergent non-volatile memories [1], novel phase change switches [2], and neuromorphic logic applications, such as adaptive oxide electronics [3]. Ferroelectric, being one such memory material, is of no exception, and its potential use has extended beyond the memory (FeRAM) [4] and actuators [5], to the gate-stack of transistors (NCFET) [6]. Although the reliability of the switchable polarization of ferroelectric materials have been studied extensively, and characterized by fatigue, imprint, endurance, etc. $[7,8]$, the stress induced leakage current (SILC), the time dependent dielectric breakdown (TDDB) and failure distribution of ferroelectric materials have only been characterized and interpreted traditionally, as if it was a classical gate oxide [9-11].

In this paper, we compare the DC and AC TDDB lifetime of ferroelectric capacitors, and find that the traditional breakdown theories for gate oxides are not sufficient to explain the AC TDDB characteristics of materials with double-well energy structure. We demonstrate that the defect generation is accelerated by the hot atom at the domain walls in ferroelectric materials generated under certain operating conditions, which explains critical TDDB experiments including the reduction of AC lifetime (Fig. $3,7,8)$, and a corresponding increase in the Weibull slope (Fig 9). We also propose the theoretical model which combines the defect generation theory of classical dielectrics and the phase change theory of ferroelectric materials (Fig. 10). The model suggests the use of pulse shaping to reduce such damage which has also been verified experimentally (Fig. 12). Finally, we discuss on the TDDB lifetime for an arbitrary pulse shape by decoupling the $\mathrm{AC}$ and the DC damage modes (Fig. 13).

\section{Device Characterization}

The structures studied are metal-ferroelectric-metal capacitors [12] with the ferroelectric materials composed of industrial, state-of-the-art $\mathrm{PZT}\left(\mathrm{Pb}(\mathrm{Zr}, \mathrm{Ti}) \mathrm{O}_{3}\right)$ with grain-size of $\sim 100 \mathrm{~nm}$ (Fig. 1c). The samples were either $70 \mathrm{~nm}$ or $450 \mathrm{~nm}$ thick. For $70 \mathrm{~nm}$ samples, the polarization-voltage (P$\mathrm{V})$ and capacitance-voltage $(\mathrm{C}-\mathrm{V})$ hysteresis characteristics are shown in Fig. 1(a,b). Conductive AFM measurements show that grain boundary (GB) regions have (i) reduced thickness $\left(\Delta \mathrm{T}_{\mathrm{OX}} \sim 10 \mathrm{~nm}\right)$, and (ii) higher leakage $\left(\mathrm{I}_{\mathrm{GB}}\right)$ due to higher pre-existing defects (Fig. 1c). To decouple the relative contributions of $\Delta \mathrm{T}_{\mathrm{OX}}$ vs. pre-existing defects in $\mathrm{I}_{\mathrm{GB}}$, we created a square trench on the oxide using an AFM tip (Fig. 1d). Even for a $20 \mathrm{~nm}$-deep trench (i.e., $\mathrm{T}_{\mathrm{OX}}=70-20=$ $50 \mathrm{~nm}$ ), the oxide leakage $\left(\mathrm{I}_{\mathrm{OX}}\right)$ remains smaller than $\mathrm{I}_{\mathrm{GB}}$ outside the trench region (Fig. 1e), confirming the importance of pre-existing defects in defining GB leakage.

\section{Lifetime is Nothing Unusual}

Once the oxides are stressed at DC, we find that the TDDB characteristics are very similar to that of a traditional oxide: the TDDB lifetime $\left(\mathrm{T}_{\mathrm{BD}}\right)$ follows a power law with voltage, with a temperature-dependent exponent which implies activated defect generation (Fig. 2a). Indeed, at relatively low frequencies, the lifetime scales perfectly with duty cycle $(d)$, indicating the absence of any relaxation (Fig. $2 b)$. However, the Weibull slope $\left(\beta_{D C}\right)$ is remarkably small $(\sim 1.5)$ for a $70 \mathrm{~nm}$ thick capacitor. We have recently demonstrated that [13] the (DC) BD-spot is always located within the GBs (Fig. 4) and the low $\beta_{D C}$ reflects 'electrical thinning' of GB region due to the large number of preexisting defects.

\section{The Puzzle of AC Lifetime and Hot Atom Damage}

Once the stress is switched to an $\mathrm{AC}$ pulse, $\mathrm{T}_{\mathrm{BD}}$ decreases dramatically, see Fig 3. For most reliability phenomena (e.g., TDDB, BTI, SILC), typically the AC lifetime is either larger (with recovery) or equal (when recovery is absent) to the derated DC lifetime of the capacitor [14-16]. In ferroelectrics, however, the AC TDDB lifetime is orders of magnitude smaller than that at DC bias (Fig. 3), indicating the presence of some additional damage 
mechanism during the AC stress. We emphasize that, here we refer to the catastrophic dielectric breakdown, which is different from fatigue (loss of switchable polarization) at AC stress[8].

A fundamental difference between a traditional dielectric and a ferroelectric material is the double-well energy landscape of the latter [17], as a function of polarization (Fig. 5). For a complete switching of the memory state, the applied voltage $\left(V_{A p p}\right)$ must exceed the coercive voltage $\left(\mathrm{V}_{\mathrm{C}}\right)$ so that the center $(\mathrm{Zr} / \mathrm{Ti})$ atom can cross the potential barrier. Hypothetically, if the AC TDDB damage is related to the repeated crossing of this barrier, the damage should be proportional to the number of the switching cycles $\left(\mathrm{N}_{\mathrm{CY}}\right)$. Fig. 6 confirms that it is indeed true, i.e., the stress induced leakage current (SILC) is determined by $\mathrm{N}_{\mathrm{CY}}$, regardless the frequency $(f)$.

At very low $f$ (at $50 \%$ d.c.), however, $\mathrm{N}_{\mathrm{CY}}$ is small as compared to the effective DC stress time. Hence we notice a shift in lifetime towards DC lifetime (Fig. 7), since the damage mode is dictated by the DC stress at such condition. To isolate the contributions of cyclic (switching) stress, we use an extremely narrow pulse train so that the DC damage is avoided. Remarkably, as the second confirmation of the HAD theory, the lifetime now simply scales linearly with frequency.

Third, to find the effect of pulse amplitude, we use different pulse shapes as in Fig. 8a. As mentioned, for repeated switching, the AC pulse must include both $\pm V_{C}$ levels (case iv, v). Otherwise, for a non-switching AC (case ii, iii), the center polarizing atom will just oscillate within a single well, effectively behaving like a traditional high-k dielectric under AC stress. For such cases (ii, iii), the lifetime is comparable with DC stress (Fig. 8b). However, as soon as the pulse becomes switching (case iv, v), there is a sharp decrease in lifetime, again relating the damage to the repeated crossing of atom across the barrier.

Theoretically, during polarization switching $\left(V_{A p p}>\right.$ $V_{C}$ ), the energy gained by the atoms at the domain walls, not only allows it to cross the central energy barrier, but also leads to an overshoot in the local polarization, until the energy is dissipated to the surroundings (Fig. 10). These transient polarization overshoots stretch the bonds around the domain walls and reduce the thermal activation energy [18] required for bond breakage (Fig. 11), and thus reduce $\mathrm{T}_{\mathrm{BD}}$.

Finally, unlike DC breakdown which is localized at the GB (with the consequence of low Weibull) [13], AC switching of atoms is inefficient at the GB edges due to the presence of defects. Rather, the switching of atoms occurs at the domain walls within the grains. Since the domain walls propagate across the grain regions during switching, the defect generation also happens along with the domain wall motion throughout the grain regions (Fig. 9a). As the preexisting defect density at grain is considerably smaller than that of the GB, we see a dramatically sharper Weibull slope for an AC stress (Fig. 9b). The results are supported by Monte Carlo simulation [13].

\section{Optimization of Device Operation}

To minimize HAD, the AC pulse must be shaped to reduce the polarization overshoot. For example, one would expect a lower HAD using a pulse with slower transitions time. This is confirmed in Fig. 12a where the lifetime increases by order of magnitude, if the pulse has an increased transition time. In fact, the slower transition of the atoms allows lesser polarization overshoots and reduced bond stretching (increased lifetime). Second, since the polarization is maximized at resonant frequencies which would lead to increased HAD (Fig. 12b), this situation must be carefully avoided.

Finally, since the DC and the switching damages are dictated by independent stress modes, one can characterize the Weibull distributions separately to find the lifetime for an arbitrary shape of the pulse $(f, d)$. The final lifetime will be governed by the prorated DC and switching lifetime based on $d$ and $f$, respectively, as shown in Fig. 13.

\section{Conclusion}

We have established a new degradation mechanism associated with the hot atom damage through a series of probing experiments, and offered an intuitively simple and consistent theory to interpret the results, and to establish TDDB lifetime of ferroelectric memories as a function of optimized bias, pulse shape, and frequency.

\section{Acknowledgement}

We acknowledge Srikanth Krishnan, John Rodriguez, and Dhanoop Varghese (TI) for useful discussions, Texas Instruments, Inc. for providing samples, AFM experimental facility, and for funding the work. We also thank Birck Nanotechnology Center for all other experimental facilities.

\section{References:}

[1] D. B. Strukov, G. S. Snider, D. R. Stewart, and R. S. Williams, Nature, vol. 453, p. 80, 2008.

[2] H. Takagi and H. Y. Hwang, Science, vol. 327, p. 1601, 2010.

[3] S. D. Ha and S. Ramanathan, J. Appl. Phys, vol. 110, p. 071101, 2011.

[4] J. F. Scott, Ferroelectric Memories: Springer Verlag, 2000.

[5] P. Muralt, J. Micromech. and Microengg, vol. 10, p. 136, 2000.

[6] S. Salahuddin and S. Datta, Nano Lett, vol. 8, p. 405, 2008.

[7] S. Sadashivan, S. Aggarwal, et al., J. Appl. Phys, vol. 83, 1998.

[8] A. K. Tagantsev, I. Stolichnov, E. L. Colla, and N. Setter, J. Appl. Phys., vol. 90, p. 1387, 2001.

[9] R. Moazzami, C. Hu, and W. H. Shepherd, IEEE Trans. Electron Devices, vol. 39, p. 2044, 1992.

[10] J. Chen, H. Chen, and J. Lee, Appl. Phys. Lett., vol. 69, p. 4011, 1996.

[11] S. Desu and I. Yoo, Integrated Ferroelectrics, vol. 3, p. 365, 1993.

[12] J. A. Rodriguez, K. Remack, K. Boku, K. R. Udayakumar, et al., IEEE Trans. Device and Materials Reliability, vol. 4, p. 436, 2004.

[13] M. Masuduzzaman, S. Xie, J. Chung, D. Varghese, et al., Appl. Phys. Lett., vol. 101, p. 153511, 2012.

[14] E. Rosenbaum and C. Hu, IEEE Electron Device Lett., vol. 12, p. 267, 1991.

[15] M. A. Alam, IEEE Int. Electron Devices Meeting, 2003, p. 14-4.

[16] W. K. Chim and Y. N. Tan, J. Appl. Phys., vol. 92, p. 6636, 2002.

[17] D. Ricinschi, C. Harnagea, C. Papusoi, L. Mitoseriu, et al, J. Physics: Condensed Matter, vol. 10, p. 477, 1998.

[18] J. W. McPherson and H. C. Mogul, J. Appl. Phys., vol. 84, pp. 1513, 1998. 
(a)

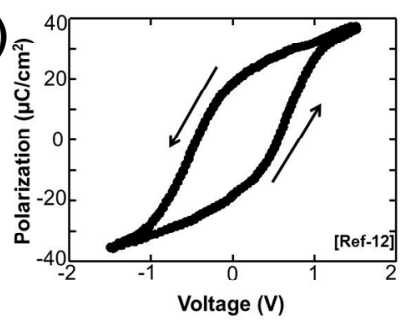

(b)

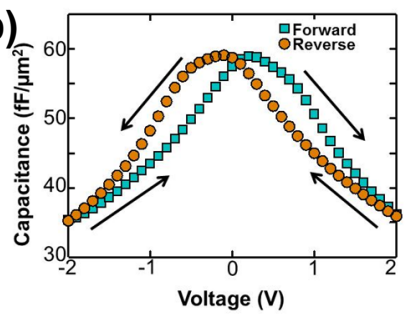

(d)

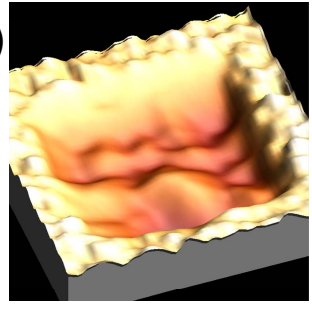

(c)

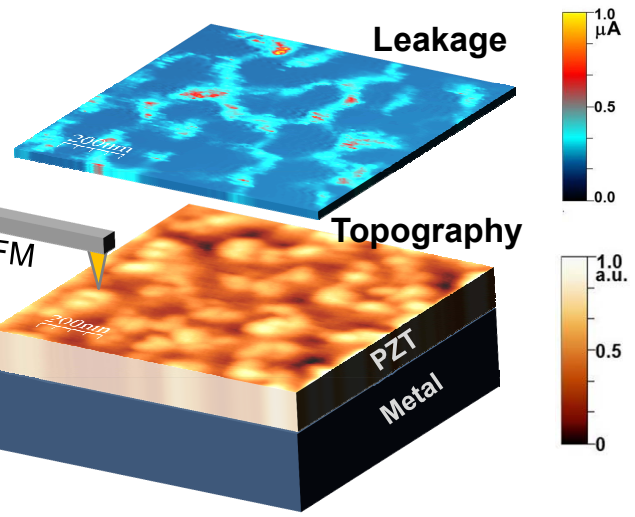

(e)
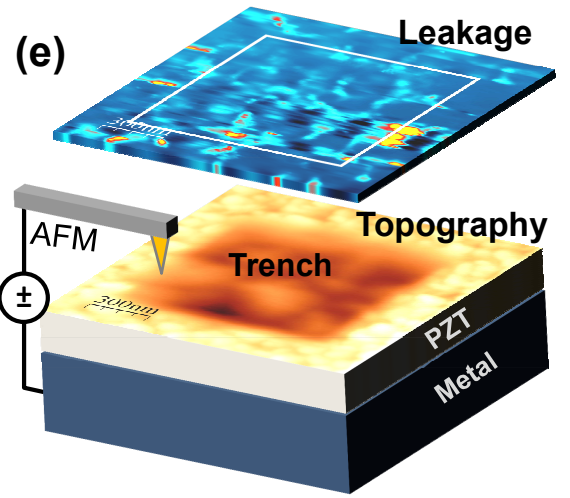

Fig. 1: Initial characterizations: (a) The polarization-voltage ( $\mathrm{P}$ V) [12] and (b) the capacitancevoltage $(\mathrm{C}-\mathrm{V})$ hysteresis curves are shown for a $70 \mathrm{~nm}$ PZT sample. (c) The topographic (bottom) and the leakage (top) maps are taken by scanning the oxide surface by a conductive AFM tip with an applied bias. The leakage current shows a clear correlation with the GB regions. (d) A $1 \mu \mathrm{m}^{2}$ trench (20nm depth) is created by repeated scanning of an AFM tip. (e) The current map shows that $\mathrm{I}_{\mathrm{GB}}$, even at the outside of the trench $(70 \mathrm{~nm}$ oxide thickness), is higher than lox (non-GB regions) within the trench (50nm oxide thickness), implying that the influence of pre-existing defects is greater than $\Delta T_{\text {ox }}$ in $G B$ leakage current.
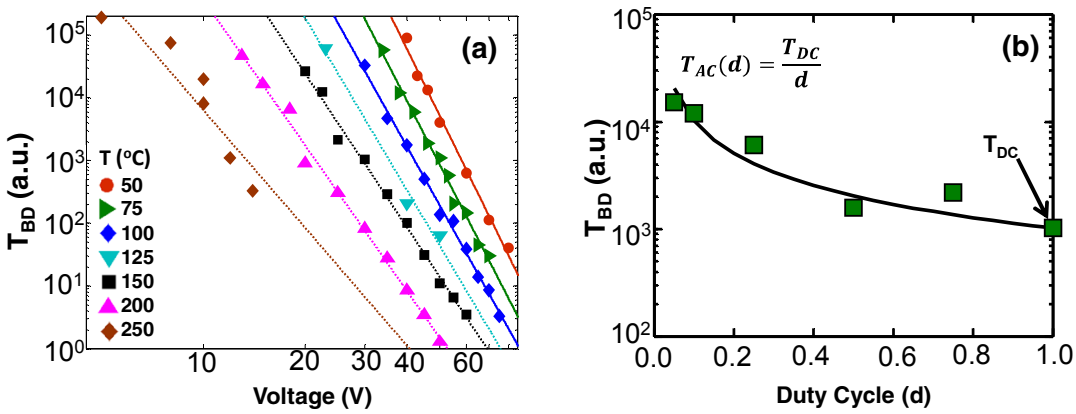

Fig. 2: (a) The lifetimes for $D C$ stress $\left(T_{O X}=450 \mathrm{~nm}\right)$ at different voltages and temperatures $(T)$ are shown. Once fitted with a power law, the voltage exponent decreases with $\mathrm{T}$, indicating thermally activated defect generation. (b) The DC and the pulsed AC lifetime are shown (symbol) as a function of duty cycle $(d)$. The line is drawn by scaling the DC lifetime with $d$.

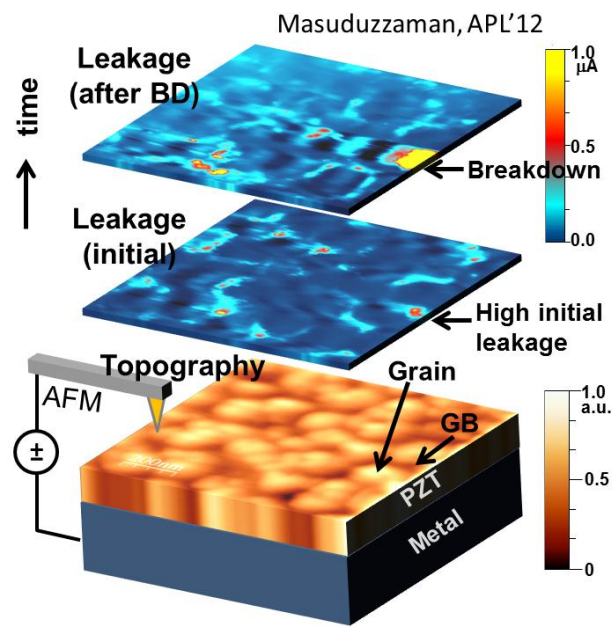

Fig. 4: Our previous study [13] shows that during DC stress, breakdown always occurs through the GB regions due to large pre-existing defects therein. This also causes very low Weibull slope at DC stress. Later (Fig. 9), we will compare this with the Weibull distribution of AC stress.

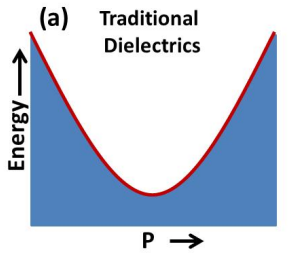

(b) Ferroelectric

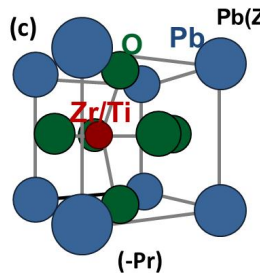

$\mathrm{Pb}(\mathrm{Zr}, \mathrm{Ti}) \mathrm{O}_{3}$

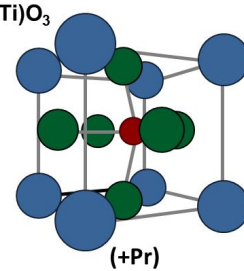

Fig. 5: Potential energy landscape as a function of Fig. 6: The leakage evolution (a proxy of polarization (P) for (a) traditional dielectric, and (b) defect generation) at a sense voltage a ferroelectric material. The later has a double- shows almost identical results as a well characteristic that enables two distinct states function of the number of stress cycles. $( \pm P r)$, as shown in (c) for PZT. The center $\mathrm{Zr} / \mathrm{Ti}$ This indicates that the internal damage is atom needs to get a certain energy (represented dictated by the switching of the by the coercive voltage in the applied potential) to polarization at this frequency range. overcome the energy hump. 


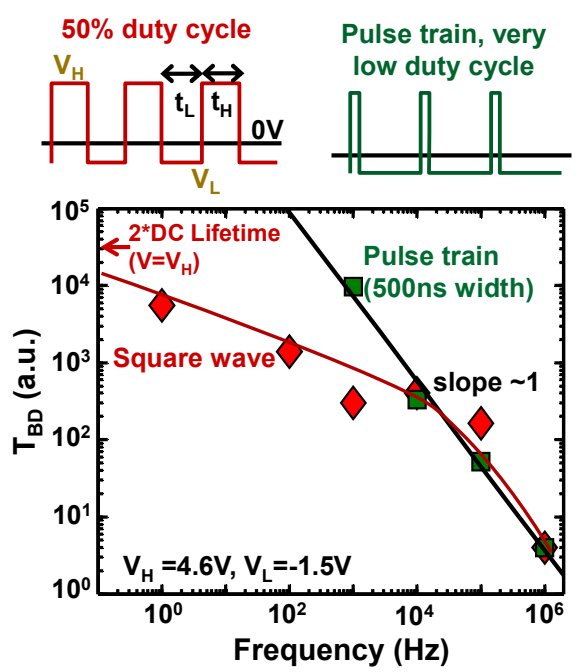

Fig. 7: Two waveform types- square wave and pulse trains- are used to isolate the $D C$ and $A C$ damage. For narrow pulse train, the lifetime scales with $f$ (only depends on the number of cycle). But for a square wave, as $f$ goes down, the DC stress becomes dominant, and the lifetime starts to merge with the derated DC lifetime.
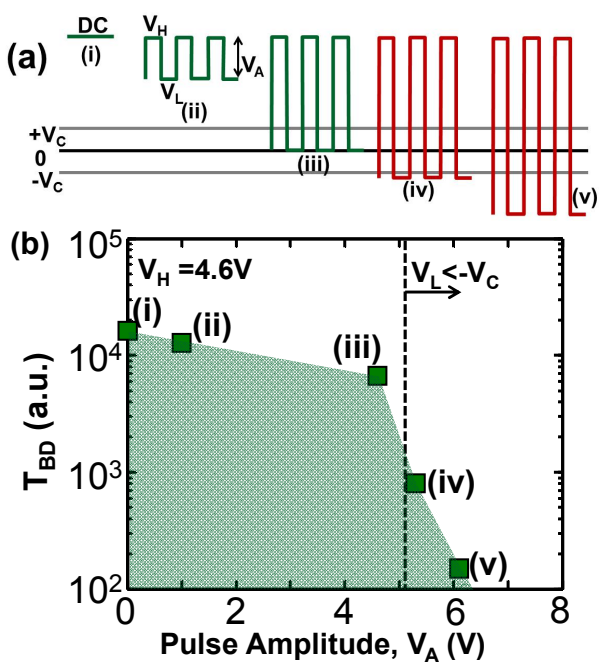

Fig. 8: For the five different waveforms (a), the corresponding lifetimes are shown in (b). Case (i) is DC. Although cases (ii)-(iii) are AC, but they do not crosses both $\pm V_{C}$, and hence can not switch the memory states, and the lifetime is comparable to DC. Only for cases (iv)-(v), the memory states switch in every cycle. The lifetime is drastically reduced in these cases.
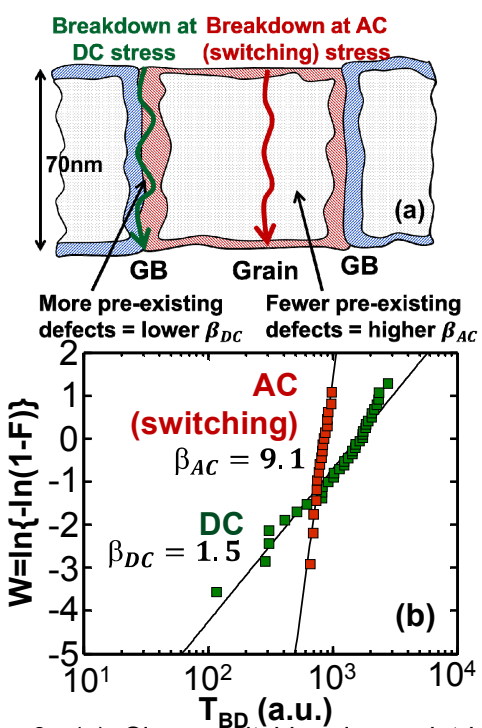

Fig. 9: (a) Since switching is an intrinsic property, the corresponding damage, and breakdown occurs throughout the grains of the material. This is in contrast to the DC stress where we have seen GB dominated breakdown (Fig. 4). (b) Such fact is reflected in the significantly sharper Weibull slope in AC breakdown. (a)

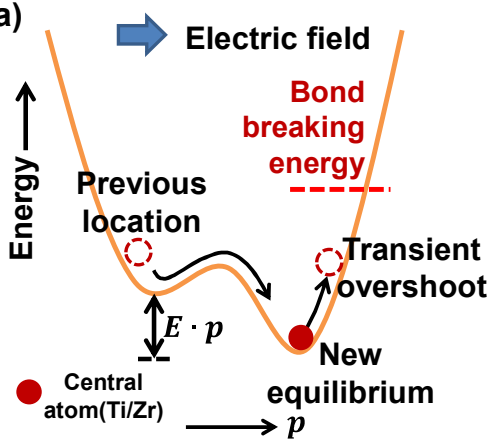

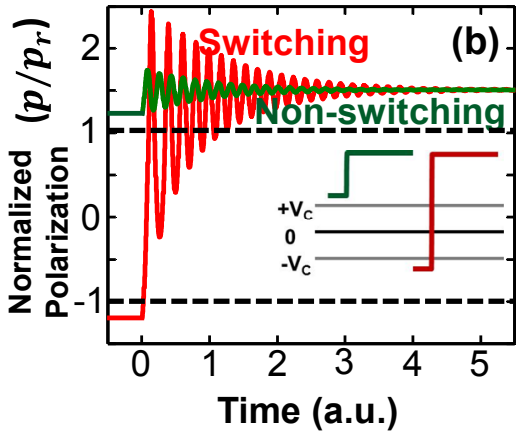

Fig. 10: (a) Once a bias $\left(>V_{C}\right)$ is applied, the center atom gets enough energy to cross the energy barrier in the middle. Once it crosses the barrier, the energy must be dissipated so that the atom settles in the new equilibrium. (b) Simulation shows that, for a switching pulse (inset, red), there is a significant transient overshoot in local polarization before this excess energy is dissipated. The activation energy for bond breakage is reduced during this time (see Eq. (2) in Fig. 11), which leads to lower AC lifetime.
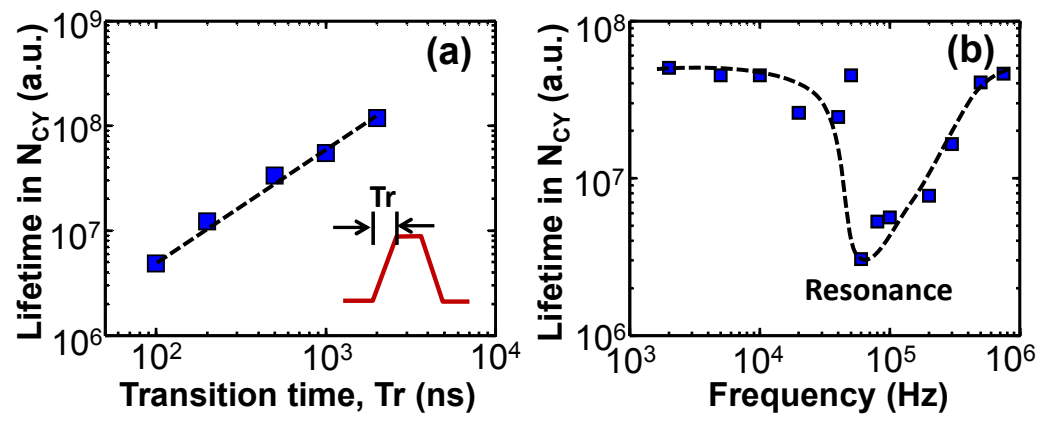

Fig. 12: (a) The AC TDDB lifetime is shown for different transition/rise time $(T r)$ of the switching pulse. As $T r$ increases, the lifetime increases significantly with a slope close to unity in a log-log plot. Clearly, the slower switching causes higher damping of the switching energy and lesser HAD results. This offers an effective control knob of the AC TDDB lifetime. (b) The polarization overshoot is maximum around resonant frequency and a notch in lifetime is observed. This condition must be avoided in the design of the operating pulse.

$$
\begin{aligned}
& \text { Breakdown } \\
& T_{B D} \propto \exp \left(E_{A}^{\prime} / k T\right) \\
& E_{A}{ }^{\prime}=E_{A 0}-\left(p_{i} \times E_{l o c}\right) \\
& \text { Polarization } \\
& M \frac{d^{2} p_{i}}{d t^{2}}+\Gamma \frac{d p_{i}}{d t}-\mathcal{F}\left(p_{i}\right)=E(t) \\
& \mathcal{F}\left(p_{i}\right)=-\frac{d}{d p_{i}}\left(\frac{a}{2} p_{i}^{2}+\frac{b}{4} p_{i}^{4}+k \nabla p_{i}\right)
\end{aligned}
$$

Fig. 11: Two sets of equations are combined to model the breakdown as a function of switching. In Eq.(1), $E_{A}$ is the activation energy for bond breakage, which reduces with the local polarization, $p_{i}$ as in Eq.(2) [18]. To solve the polarization dynamics for an applied external field $(E(t))$, Eq.(3) is used, where $\mathcal{F}\left(p_{i}\right)$ is the internal field originating from the double-well potential profile, characterized by $a$, $b, k[17] . M, \Gamma$ are the inertial and damping coefficients.

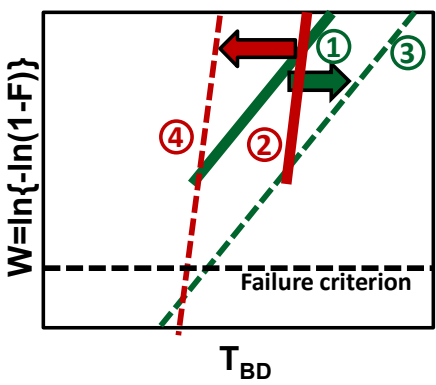

Fig. 13: The scaling of lifetime with duty cycle $(d)$ for DC stress (Fig. 2b), and with frequency $(f)$ for switching stress (Fig. 7), allow us to calculate the lifetime for a input pulse with arbitrary $d_{O P}$ and $f_{O P}$. Lines (3) and (4) are calculated by shifting lines (1) and (2) by $\ln \left(d_{O P}\right)$ and $\ln \left(f_{\text {Meas }} / f_{O P}\right)$, respectively. Based on the fail-fraction criteria, the ultimate lifetime could be determined by both of the DC and the switching stress, and needs to be optimized accordingly. 